\title{
Barrett's oesophagus: where are we now?
}

\author{
S. Wijetunge ${ }^{1}$, R. Kotakadeniya ${ }^{2}$ \\ 1 Department of Pathology, Faculty of Medicine, University of Peradeniya, Sri Lanka \\ 2 Department of Surgery, Faculty of Medicine, University of Peradeniya, Sri Lanka
}

Key words: Barrett's oesophagus; columnar lined oesophagus; gastro-oesophageal reflux disease; oesophageal adenocarcinoma.

\begin{abstract}
With the high prevalence of gastro-oesophageal reflux disease, the incidence of oesophageal adenocarcinoma is increasing in many parts of the world, particularly in the West. Therefore, recognition of Barrett's oesophagus, its premalignant stage, has become important in the management of gastro-oesophageal reflux disease. This review focuses on the evolution of knowledge since the first description of the columnar lined oesophagus, the current status and controversies surrounding Barrett's oesophagus, and problems associated with its management.
\end{abstract}

\section{Introduction}

The incidence of gastro-oesophageal reflux disease (GORD) and its complications has been rising in many parts of the world, particularly in developed Western populations, similar to other life-style related diseases such as cardiovascular diseases, type- 2 diabetes and metabolic syndrome [1,2]. Moreover, in the long- term GORD is known to produce oesophageal adenocarcinoma, through its premalignant stage, Barrett's oesophagus.

An increase of epidemic proportions has been observed in the USA, where from the mid 1970's the incidence of oesophageal adenocarcinoma has steadily risen to surpass the incidence of oesophageal squamous cell carcinoma; the relative incidence of oesophageal adenocarcinoma (adenocarcinoma per squamous cell carcinoma $\mathrm{x} 100$ ) in the USA has risen from $23.9 \%$

Correspondence: S. Wijetunge

E-mail: suwijetunge@gmail.com during 1976-1987 to $158 \%$ in 2002 [2]. In developed Asian countries such as Singapore, Japan and Taiwan, although squamous cell carcinoma is still the most common type of oesophageal malignancy, adenocarcinoma has shown an increasing trend [3]. In Sri Lanka, according to the National Cancer Control Programme data, the relative incidence of oesophageal adenocarcinoma has risen from $8 \%$ to $14 \%$ during the period 2000 to 2005 [3]. At our institution, the relative incidence of oesophageal adenocarcinoma has risen from $1.8 \%$ during $1991-1993$ to $11.6 \%$ during 2012-2013 [4].

The sequence of events from GORD to oesophageal adenocarcinoma are as follows:

GORD $\longrightarrow$ Barrett's oesophagus $\longrightarrow$ Dysplasia Adenocarcinoma

However, despite the high prevalence, the lack of clear understanding has resulted in multiple ways of management of GORD and Barrett's oesophagus. Here, we take a closer look at the changing knowledge of Barrett's oesophagus since its description in the 1950's and discuss the present status of the disease.

\section{Evolution of the concept of Barrett's oesophagus}

Around the mid twentieth century, some surgeons observed the presence of a columnar lined tubular structure distal to the squamous lined oesophagus, and there were descriptions of ulcers in this area. In 1950, Norman Barrett, a renowned British surgeon, defined the oesophagus as the part of lower oesophagus which is lined by squamous epithelium and described the distal columnar lined structure as tubular stomach which had been pulled up due to a congenitally shortened oesophagus [5]. However, in 1953, Allison and 
Johnstone identified that the region which Barrett described as "tubular stomach" did not have a peritoneal lining and gastric type musculature but comprised oesophageal type submucosal glands [6]. These authors suggested that Barrett's description of the "tubular stomach" was part of the oesophagus lined by "gastric type membrane" [6]. In 1957 Barrett acknowledged that Allison and Johnstone were correct and agreed to the term columnar lined lower oesophagus [7]. However, Barrett, Allison and Johnstone yet believed that the columnar lining in the oesophagus was congenital. Later, Barrett began to believe less in his congenital theory and stated that "if the cardiac valve of a normal person were to become incompetent and if the lower oesophagus were, as a result, to be bathed for a long time by digestive gastric juice, the squamous epithelium could be eaten away and totally replaced by columnar cells" [7]. Subsequent studies by Moersch et al. in 1954 suggested that the columnar lined oesophagus (CLO) was metaplastic, and was due to gastro-oesophageal reflux $[8,9]$; this was confirmed by Bremner et al. in 1970 [9].

In 1961, Hayward described that the normal gastrooesophageal junctional region was lined by a nonspecialized mucin secreting columnar epithelium he termed as junctional mucosa, and hence, it was normal to have the lower $1-2 \mathrm{~cm}$ of the oesophagus lined by glandular mucosa [7]. As a result, presence of a columnar lined oesophagus (CLO) up to $2 \mathrm{~cm}$ was regarded as normal and a length of atleast $3 \mathrm{~cm}$ above the gastro-oesophageal junction was required to make a diagnosis of CLO. This was, until the concept of short segment Barrett's oesophagus was put forward based on work by Spechler et al. in the 1990's [11]. Notwithstanding the work by Spechler, the $3 \mathrm{~cm}$ rule and the use of the term junctional mucosa to describe the cardiac mucosa found at the lower oesophagus are still used in practice by some surgeons and pathologists.

Since the late 1950 's CLO was commonly referred to as Barrett's oesophagus without an indication of prognostic significance, a practice that is still continued by some. Paull et al, in 1976, described the histology of CLO as fundic (present term oxyntocardiac mucosa), junctional (cardiac mucosa) and specialized type (intestinal metaplasia) [12]. The significance of these epithelial types was not evident until Haggit suggested that intestinal type metaplasia in the CLO increased the risk of adenocarcinoma, an observation which was later confirmed by many others [13 -16]. Afterward, the terms CLO and Barrett's oesophagus were not recommended to be used synonymously instead, Barrett's oesophagus was used to define the CLO which meant that this part of the oesophagus bore a significantly increased risk of progression to adenocarcinoma. Hence, the Practice Parameters Committee of the American College of Gastroenterologists, in its 1998 statement, defined Barrett's oesophagus as the presence of endoscopically visible CLO within the oesophagus, which on biopsy, was confirmed to have intestinal metaplasia [17).

\section{Where are we now?}

It is now known that the normal oesophagus is entirely lined by squamous epithelium and the presence of columnar lining in any part of its length indicates metaplastic changes due to reflux induced damage. The CLO may contain three types of metaplastic glandular tissue: cardiac mucosa (CM)- composed of non specialized mucus secreting columnar cells, oxyntocardiac mucosa (OCM)- a mixture of non specialized cells and oxyntic cells and intestinal metaplasia (IM)-composed of goblet cells.

\section{Definition of Barrett's oesophagus}

It is now universally accepted that, of the three metaplastic glandular tissue types in the CLO, intestinal metaplasia - the most unstable, carries a significant risk of progression to adenocarcinoma. Early studies have reported risk of adenocarcinoma levels as high as $2-4 \%$ per year. However, currently, the widely accepted risk level is $0.5 \%$ per year ( 1 in 200 patients with Barrett's oesophagus will develop oesophageal adenocarcinoma each year). This data was derived from a large metaanalysis after adjusting for possible confounding factors $[18,19]$.

Currently, there is a belief that metaplastic cardiac mucosa, per se, without intestinal metaplasia can also predispose to malignancy. Such belief is based on similar molecular changes seen in intestinal metaplasia as is in cardiac mucosa. Furthermore, there are reports of 
gastro-oesophageal resection specimens with malignancy without intestinal metaplasia in the adjacent non-neoplastic tissue [18]. However, the role of these shared molecular changes in the neoplastic progression is not known. It is also known that with malignant progression intestinal metaplasia often becomes replaced by dysplastic or neoplastic tissue. Furthermore, even if CM carries a risk for development of malignancy, the magnitude of the risk is not known [18]. Therefore, in the latest 2011 American Gastroenterologist Association (AGA) position statement on Barrett's oesophagus, presence of cardiac mucosa lined oesophagus without intestinal metaplasia has not been considered a significant risk factor for malignancy owing to insufficient evidence [20]. The definition of Barrett's oesophagus in the 2011 AGA position statement is as follows:

"Barrett's oesophagus is the condition in which any extent of metaplastic columnar epithelium that predisposes to cancer development replaces the stratified squamous epithelium that normally lines the distal oesophagus. Presently, intestinal metaplasia is required for the diagnosis of Barrett's oesophagus because intestinal metaplasia is the only type of oesophageal columnar epithelium that clearly predisposes to malignancy."

By contrast, the 2013 British Society of Gasteroenterologist (BSG) guidelines have acknowledged that the evidence favouring cardiac mucosa alone as a significant risk factor for development of malignancy is weak, nevertheless stating that "the presence of intestinal metaplasia is not a prerequisite for the definition of Barrett's oesophagus, but should be taken into account when deciding on clinical management" [21].

Treating CM without IM, as a premalignant condition despite the lack of awareness of its real risk can have the following consequences: 1) $\mathrm{CM}$ is an extremely common finding in CLO and if all those having CM are to undergo intense surveillance as for a premalignant condition it will add a huge workload and cost to the health care services. 2) Labeling a patient as having a premalignant condition can cause significant psychological stress and have a considerable economic and social impact on the individual.
Of the three metaplastic epithelial types in the CLO, oxyntocardiac mucosa (OCM) is the most stable epithelial type and is not associated with malignant progression [22]. According to the authors' experience, in Sri Lanka where the prevalence of oesophageal adenocarcinom is low, compared to the West, oxyntocardiac mucosa is very common in the columnar line mucosa and intestinal metaplasia is rather uncommon (unpublished data).

\section{Where is the gastro-oesophageal junction?}

According to the 2011 AGA position statement "The diagnosis of Barrett's oesophagus can be suspected when, during endoscopic examination, columnar epithelium is observed to extend above the gastroesophageal junction (GOJ) into the tubular oesophagus" [20], but the reliability of endoscopic identification of the GOJ remains unclear.

In the West, the most commonly used endoscopic landmark for the GOJ is the proximal limit of the gastric rugal folds. However, the proximal limit of gastric rugal folds are subjected to change constantly with respiration, gut motility and the degree of abdominal distension [18]. Furthermore, Chandrasoma et al. have proposed that when the lower oesophageal sphincter is damaged, which is a constant observation in GORD, the distal intra-abdominal part of the oesophagus dilates due to negative pressure and assumes gastric like contours and even develops rugal folds. Also, when this dilated part of oesophagus becomes lined by metaplastic columnar epithelium as seen on endoscopy, it becomes practically impossible to differentiate lower oesophagus from the proximal stomach; he called this the dilated distal oesophagus (DDO) [23]. Accordingly, the landmark of proximal limit of the rugal folds becomes even more unreliable in patients with GORD.

In Asia, especially East Asia, the distal limit of the palisade veins located in the distal oesophageal mucosa is commonly used as the landmark for GOJ [18]. However, the anatomical relationship of these palisade veins with the GOJ has not been proven and visualization of these veins with precision becomes difficult with oesophagitis [18]. This means that the two most commonly used endoscopic landmarks for the GOJ in the world are flawed. While acknowledging the 
limitations of currently used landmarks, for want of a better landmark, both AGA and BSG guidelines recommend continued use of proximal limits of the gastric rugal folds as the endoscopic landmark of GOJ $[20,21]$.

\section{Short segment Barrett or intestinal metaplasia of cardia?}

According to the widely held belief, short segment Barrett's and IM of the cardia are two disease entities with different aetiopathogeneses, i.e., short segment Barrett is due to GORD and IM of cardia is due to Helicobacter pylori infection [24]. Given the unreliability of the endoscopic landmarks of the GOJ, it is difficult for the endoscopist to reliably differentiate short segment CLO from the proximal stomach. Histologically, oesophageal origin of a mucosal biopsy can be reliably identified by the presence of submucosal glands or their ducts. If IM is seen in the presence of these structures the diagnosis of Barrett's oesophagus may be made reliably. Since studies have shown that such indicators of oesophageal origin are present in only about $10-15 \%$ of cases, reliable histological diagnosis of short segment Barrett's can be made only occasionally [21]. The other useful indicators of oesophageal origin of a biopsy are multilayered epithelium at the squamo-columnar junction and squamous islands within the glandular tissue [25]. BSG 2013 guidelines suggest that "diagnosis of Barrett's oesophagus should take into account the degree of confidence based on a combined analysis of endoscopic and histopathological criteria" [21]. Considering the unreliability of the endoscopic landmarks of GOJ in GORD patients, the practical relevance of this statement in a diagnosis of short segment Barrett becomes questionable.

\section{Could the gastro-oesophageal junction be defined histologically?}

The first attempt of histological definition of GOJ was made by Norman Barrett when he defined the oesophagus as the part lined by squamous epithelium and the stomach as the part lined by columnar epithelium [5]. Later, it became understood that this definition, which may hold true for the normal oesophagus, cannot be applied to reflux damaged oesophagus.

Recently, Chandrasoma et al. proposed that the cardiac mucosa, which is believed to be the normal mucosal lining of the proximal stomach (cardia), is not a normal epithelium but a metaplastic epithelium that lines the lower oesophagus due to reflux induced damage [2530]. According to him the erroneous belief of CM lining the proximal stomach is due to the lack of awareness of the existence of a dilated distal oesophagus which we recognize as the cardia [23]. The true proximal stomach is lined by oxyntic mucosa. According to this hypothesis, GOJ can be histologically defined, and in a normal person without reflux damage, it is the junction between the squamous epithelium and oxyntic mucosa. In those with reflux induced damage GOJ is the junction between CM and oxyntic mucosa or the OCM and oxyntic mucosa. He further proposed the term squamooxyntic gap to histologically define the CLO. Accordingly, the columnar epithelial types (CM, OCM and IM) between the squamous epithelium and oxyntic mucosa are metaplastic due to reflux induced damage and are oesophageal in origin [31]. Based on this theory, the presence of $\mathrm{CM}$ in a biopsy always indicates oesophageal origin and presence of IM admixed with CM indicates Barrett's oesophagus; thus the term IM of cardia becomes obsolete. Furthermore, adenocarcinoma arising from the GOJ and cardia are oesophageal in origin and are due to GORD. However, this theory has not yet gained wide acceptance and is practiced only in a few tertiary care centres treating GORD in the USA and in Europe.

Epidemiological studies have shown that oesophageal, junctional and proximal gastric/cardia adenocarcinoma demonstrate a similar rising pattern, whereas distal gastric carcinoma shows a declining incidence [32, 33]. Clinical features of adenocarcinoma around the GOJ have been shown to be similar and different to distal gastric carcinoma [34, 35]. Furthermore, H. pylori infection, which is the most common aetiological agent for distal gastric carcinoma has been shown not to be associated with cardia and junctional adenocarcinoma [36-38]. These observations are in favour of the view of a common aetiopathogenesis for adenocarcinoma arising around the "GOJ". 


\section{Does the length of CLO matter?}

Traditionally, Barrett's oesophagus was divided into short segment Barrett $(<3 \mathrm{~cm})$ and long segment Barrett. Accordingly, adenocarcinoma of the gastrooesophageal junctional region is believed to arise from the short segment Barrett [11]. However, both short segment and long segment Barrett are managed similarly $[20,21]$.

The prevalence of IM appears to increase with the length of the CLO $[18,21,31]$. In one series all CLO more than $5 \mathrm{~cm}$ in length had IM [30]. When IM is not evenly distributed it is more likely to be found in the most proximal regions in the CLO [22,31]. Therefore, when obtaining biopsies from CLO it is important to include biopsies from the most proximal regions of CLO, preferably, straddling the squamo-columnar junction. The cancer risk also appears to increase with the extent of the columnar metaplasia. Therefore, both AGA and BSG guidelines recommend systematic recording of the circumferential extent and maximum extent of metaplasia in endoscopic reports using a methodology such as the Prague criteria [20,21,39].

\section{Natural history of Barrett's oesophagus}

As mentioned earlier the presence of IM in the oesophagus carries a $0.5 \%$ per year risk of progression to adenocarcinoma through dysplasia. Therefore, in patients identified to have Barrett's oesophagus surveillance for dysplasia is recommended [20, 21]. Histologically, dysplasia is graded as low grade dysplasia and high grade dysplasia. A meta-analysis in 2008 reported the risk of adenocarcinoma in those who had high grade dysplasia (without prevalent cancer at the time of diagnosis) as $6.6 \%$ per year [40]. The risk of cancer with low grade dysplasia has not been defined with certainty. Some studies have shown no increased risk than those who have IM alone while others have shown an increased risk [18]. The main reason for this discrepancy is high inter-observer variability, hence low reproducibility, of histological diagnosis of low grade dysplasia [20, 41, 42]. The difficulty in differentiating between reactive changes associated with inflammation and low grade dysplasia is the main cause for this. It has also been widely observed that those cases with Barrett's oesophagus with low grade dysplasia do not often show dysplastic changes in subsequent biopsies. It is not clear whether this is because low grade dysplasia is commonly reversible or there is an over-diagnosis of reactive changes. Another problem with dysplasia assessment is the diagnosis of "indefinite for dysplasia" with which further management options become questionable. Although use of a molecular marker, which correctly predicts the risk of malignant progression, seems to be the answer to these problems, there are no validated biomarkers available to assess the progression of malignancy in Barrett's oesophagus. Therefore, despite the mentioned limitations with the histology the general recommendation for surveillance of Barrett's oesophagus is endoscopic and histological assessment [20, 21]. To minimize inter-observer variation it is recommended that all suspected dysplasia should be confirmed by a second pathologist $[20,21]$.

Although it has been proven that IM in CLO increases the risk of progression to malignancy, whether increased surveillance of Barrett's oesophagus reduces the morbidity and mortality is not proven. The general recommendation by popular guidelines is to perform surveillance endoscopy and biopsy at intervals of 3 to 5 years for patients who have Barrett's oesophagus without dysplasia, 6 to 12 monthly for those found to have low-grade dysplasia, and every 3 months for patients with high-grade dysplasia who receive no invasive therapy (the exact time periods vary in different guidelines) [18]. Both AGA and BSG guidelines recommend "The Seattle protocol" for obtaining surveillance biopsies, i.e., 4 quadrant biopsies from every $1-2 \mathrm{~cm}$ of the CLO ( $1 \mathrm{~cm}$ biopsies if dysplasia is suspected). In addition, areas of mucosal irregularity (nodules, masses, ulceration etc), which are more likely to be associated with dysplasia, should be sampled separately [20,21]. Studies have proven that such rigorous surveillance protocols increase the detection of high grade dysplasia and invasive malignancy $[43,44]$.

\section{Conclusion}

More than 60 years after the beginning of the discussion on Barrett's oesophagus, there remains considerable confusion and controversy. There is no universal agreement on fundamental issues like the definition of Barrett's oesophagus, normal anatomy and histology of 
the GOJ. It is likely that the very high prevalence of gastro-oesophgeal reflux related changes in the GOJ, especially in the Western populations where most studies have been conducted, have blurred the line between normal and abnormal and contributed to this confusion.

\section{References}

1. Blot WJ, Devasa SS, Kneller RW, Fraumeni JF. Continued climb in rates of oesophageal adenocarcinoma: An update. JAMA 1993:270:1320.

2. Cancer incidence in five continents volume IX. International Agency for Research on Cancer, WHO and International Association of Cancer Registries (http://www.iarc.fr)

3. Wijetunge S, Perera SN. Changing trends of oesophageal adenocarcinoma and cardia carcinoma incidence in Asia: review of literature and analysis. Journal of Diagnostic Pathology 2011; 6: 17-27.

4. Rankothkumbura JP, Weerasinghe GS, Wijetunge S, Kotakadeniya HMSRB, Nallaperuma OL. Increasing trends of oesophageal adenocarcinoma in a sample of patients from Teaching Hospital, Peradeniya. Proceedings of 36th annual academic sessions, Kandy Society of Medicine 2014.

5. Barrett NR. Chronic peptic ulcer of oesophagus and "oesophagitis". Br J Surg 1950; 38: 175 - 182.

6. Allison PR, Johnstone AS. The esophagus lined with gastric mucus membrane. Thorax 1953; 8: 87-101.

7. Barrett M. The lower esophagus lined by columnar epithelium. Surgery 1957; 41:881-894.

8. Moersch R, Ellis F, McDonald JR. Pathologic changes occurring in severe reflux esophagitis. Surg Gynecol Obstet 1959; 108:476-484.

9. Bremner CG, Lynch VP, Ellis HF. Barrett's esophagus: congenital or acquired? An experimental study of esophageal mucosal regeneration in the dog. Surgery 1970; 68:209-216.

10. Hayward J. The lower end of the oesophagus. Thorax. 1961; 16:36-41.

11. Spechler SJ, Zeroogian JM, Antonioli DA, et al. Prevalence of metaplasia at the gastroesophageal junction. Lancet 1994; 344: 1533 - 1536.

12. Paull A, Trier JS, Dalton MD, Camp RC, et al. The histologic spectrum of Barrett's esophagus. N Eng J Med. 1976; 295: 476-480.
13. Haggitt RC, Tryzelaar J, Ellis FH, Colcher $\mathrm{H}$. Adenocarcinoma complicating columnar epitheliumlined (Barrett's) esophagus. Am J Clin Pathol 1978; 70:1-5.

14. Skinner DB, Walther BC, Riddell RH, Schmidt H, Iascone C, De- Meester TR. Barrett's esophagus. Comparison of benign and malignant cases. Ann Surg 1983; 198:554-65.

15. Haggitt RC Barrett's esophagus, dysplasm and adenocarcinoma. Hum Pathol 1994; 25:982-993.

16. Chandrasoma P, Wijetunge S, DeMeester S, Ma Y, Hagen J, MD, Zamis L, DeMeester T. Columnar-lined oesophagus without intestinal metaplasia has no proven risk of adenocarcinoma. Am J Surg Pathol 2012; 36 (1): 1 $-7$

17. Sampliner RE. Practice guidelines on the diagnosis, surveillance and therapy of Barrett's esophagus. The Practice Parameters Committee of the American College of Gastroenterology. Am J Gastroenterol 1998; 93:10281032.

18. Spechler SJ, Sharma P, Souza RF, Inadomi JM, et al. American Gastroenterological Association technical review on the management of Barrett's esophagus. Gastroenterology 2011;140:18-52.

19. Yousef F, Cardwell C, Cantwell MM, Galway K, et al. The incidence of esophageal cancer and high-grade dysplasia in Barrett's esophagus: a systematic review and metaanalysis. Am J Epidemiol. 2008; 168:237-249.

20. The AGA Institute Medical Position Panel. American Gastroenterological Association Medical Position Statement on the Management of Barrett's Esophagus. Gastroenterology 2011;140:1084-1091

21. Fitzgerald R, di Pietro M,Ragunath K, Ang Y,Kang JY,Watson P et.al. British Society of Gastroenterology guidelines on the diagnosis and management of Barrett's oesophagus. Gut. 2014;63:7-42.

22. Chandrasoma PT, Der R, Dalton P, et al. Distribution and significance of epithelial types in columnar-lined esophagus. Am J Surg Pathol. 2001; 25: 1188-93.

23. Chandrasoma P, Wijetunge S, Ma Y, DeMeester, S, Hagen J, DeMeester T. The Dilated Distal Esophagus: A New Entity That Is the Pathologic Basis of Early Gastroesophageal Reflux Disease. American Journal of Surgical Pathology 2011; 35: 1873 - 1881.

24.Odze R.D. Unraveling the Mystery of the Gastroesophageal Junction: A Pathologist's Perspective. Am J Gastroenterol 2005; 100:1853-1867.

25. Chandrasoma PT. Diagnostic atlas of Gastro-oesophageal Reflux disease. Amsterdam. Academic Press. 2007 
26. Chandrasoma P, Makarewicz K, Wickramasinghe K, et al. A proposal for a new validated histological definition of the gastro-oesophageal junction. Human Pathol. 2006; 37 : $40-47$.

27. Der R, Tsao-Wei DD, DeMeester T, Jeffrey P, et al. Carditis - A manifestation of gastroesophageal reflux disease. Am J Surg Pathol. 2001; 25: 245-252.

28. Oberg S, Peters JH, DeMeester T et. al. Inflammation and specilized intestinal metaplasia of cardia mucosa is a manifestation of gastro-oesophageal reflux disease. Ann Surg 1994; 226: 522-532

29. Chandrasoma PT, Der R, Ma Y et al. Histology of the gastro-oesophageal junction: an autopsy study. Am J Surg Pathol 2000; 24: 402-9

30. Chandrasoma PT, Der R, Ma Y, et al. Histologic classification of patients based on mapping biopsies of the gastroesophageal junction. Am J Surg Pathol . 2003; 27: 929-36.

31. Chandrasoma PT, Wijetunge S, DeMeester SR et al. The Histologic Squamo-oxyntic Gap: An Accurate and Reproducible Diagnostic Marker of Gastroesophageal Reflux Disease. Am J Surg Pathol. 2010; 34: 1574-1581.

32. Brown LM, Devesa SS. Epidemiologic trend in esophageal and gastric cancer in the United States. Surg Oncol Clin NAm 2002;11:235-56.

33. Devasa SS, Blot WJ, Fraumeni JF Jr. Changing patterns of in the incidence of oesophageal and gastric carcinoma in the United States. Cancer 1998; 83:2049-2053.

34. Wijnhoven BPL, Siersema PD, Hop WCJ, van Dekken H. Adenocarcinoma of the distal oesophagus and gastric cardia are one clinical entity. British Journal of Surgery; 1999; 86: 529-535.

35. Leers JM, DeMeester SR, Chan N, et al. Clinical characteristics, biologic behavior, and survival after esophagectomy are similar for adenocarcinoma of the gastro-oesophageal junction and the distal esophagus. Journal of Thorasic and Cardiovascular Surgery. 2009; 138:594-602.
36. Kamangar F , Dawsey SM, Blaser MJ, Perez-Perez GI, Pietinen P, Newschaffer CJ, et.al. Opposing risks of gastric cardia and noncardia gastric adenocarcinoma associated with Helicobacter pylori seropositivity. Journal of the National Cancer Institute, 2006; 98:14451452 .

37. Wijetunge S, Ma Y, Hagen J, DeMeester S, DeMeester T, Chandrasoma PT. Association of adenocarcinoma of the oesophagus, "gastro -oesophageal junction" and "gastric cardia" with gastric pathology. American Journal of Surgical Pathology. 2010; 34: 1521-1527.

38. Chandrasoma P, Wickramasinghe K, Ma Y, DeMeester T. Adenocarcinoma of distal esophagus and "gastric cardia" are predominantly oesophageal carcinoma. American Journal of Surgical Pathology 2007; 31: 569-575.

39. Sharma P, Dent J, Armstrong D, Bregman JJGH et.al. The Development and Validation of an Endoscopic Grading System for Barrett's Esophagus: The Prague C \& M Criteria. Gastroenterology 2006;131:1392-1399.

40. Rastogi A, Puli S, El-Serag HB, et al. Incidence of esophageal adenocarcinoma in patients with Barrett's esophagus and high-grade dysplasia: a meta-analysis. Gastrointest Endosc. 2008; 67:394-398.

41. Curvers WL, Kate FJT, Krishnadath KK,Visser M, et.al. Low-Grade Dysplasia in Barrett's Esophagus: Overdiagnosed and Underestimated. Am J Gastroenterol 2010;105:1523-1530.

42. Skacel M, Petras RE, Gramlich TL, Sigel JE, et.al. The diagnosis of low-grade dysplasia in Barrett's esophagus and its implications for disease progression. The American Journal of Gastroenterology 2000; 95:3383-3387.

43. Fitzgerald RC, Saeed IT, Khoo D, Farthing MJG, et al. Rigorous surveillance protocol increases detection of curable cancers associated with Barrett's esophagus. Dig Dis Sci. 2001; 46:1892-1898.

44. Abela JE, Going JJ, Mackenzie JF, McKernan M, O'Mahoney S, Stuart RC.Systematic four-quadrant biopsy detects Barrett's dysplasia in more patients than nonsystematic biopsy. Am J Gastroenterol 2008; 103:850-5. 